\title{
Editorial
}

\section{Cognitive Equilibirum: A New Paradigm of Decision Making?}

Human decision making and its description, modeling and computer support are currently undergoing fundamental rethinking, if not scientific revolution. We are beginning to realize how little, if anything, have we learned about human decision making so far.

Mostly, we have imposed a mathematical artifact, both simple and simple-minded in its design, on the rich, natural, self-organizing and knowledge-producing processes of individual and social decision making, without even attempting for its deeper understanding.

The very triviality of this 'paradigm' makes it self-evident and thus beyond criticism. Define a set (given, closed and/or convex) of fixed, well-defined alternatives, assign a number to each of its components according to a more or less complex (utility, preference) function or rule, then search (algorithmically) and identify the alternative(s) receiving the largest number. Label this mechanistic measurement and search routine as Decision Making and its perpetrator as The Decision Maker (DM). Base most of your economic, financial and psychological theories on this remarkable insight into the "nature of things.'

Softening things a bit, by invoking multiple functions (or criteria) instead of a single one, and assigning a vector of numbers rather than one, helps, but does not cure the chronic sense inadequacy and inferiority: certainly, the world of human systems cannot be as simple as that? Certainly, one does not extract enough satisfaction (professional or ethical) from draping the above 'paradigm' in formulas,

IOS

Human Systems Management 8 (1989) 185-188 logical rules and computer codes. What if this 'emperor' has no clothes, too?

Interest in interactive decision support systems (DSS) calls for a better descriptive understanding of the processes of human decision making: how do humans actually go about 'producing' (or constructing) their decisions? It is now proposed that no aspect or dimension of the decision-making process should be fixed a priori: criteria, alternatives, constraints, measurements, evaluations and representations - all are in continuous flux, all change and all are repeatedly reformulated in search for patterns, wholeness, harmony or cognitive equilibria.

A decision is increasingly recognized and accepted as emergent 'harmonious' pattern, properly balancing all decisional components. Recent advances in neuroscience, cognitive sciences and the associated psychological data show clearly that the conventional wisdom of so called 'rationality' is incorrect [1]. Human decision making and problem solving process is determined by the way neural networks are structured as a whole: as a spontaneously wired and continually re-wired self-organizing 'market' of repeatedly propagated patterns of formulation, re-formulation and re-formulation and reformulation ...

We now hold the means of explaining why people remain so stubbornly and extravagantly irrational, ignoring logic, maximization principles and even self-interest, so often postulated by conventional models. The answer appears to be strikingly simple: humans do not maximize functions, but search for recognizable patterns. Decision making is not about maximizing some components subject to assumed levels of other components; it is about stable patterns of harmony among all components. 
So, conventional rules, algorithms, computations and logic play no causative role in this grand re-conceptualization. They are only surface-like structural manifestations of the underlying organization of generative processes, as for example in autopoietic (self-producing) networks [4].

Margolis [1] goes even further by insisting that all thinking and judgment can be 'reduced' to pattern recognition (or 'P-cognition'). Human thinking is not to be modeled by logical rules and calculations, but through application (or even matching) of 'habits of mind' (patterns) prompted by specific contexts. This remarkable paradigmatic shift should infuse new and unprecedented vigor into the fields of MCDM, DSS, AI and perhaps even lead to their integration.

Human beings are irrational because their neural networks, productive engines of their decisions, have evolved in a particular (P-cognitive) way that is incompatible with the simplifying constructs of 'maximization,' even though many patterns of harmony could reveal characteristics of 'as-if' maximization in hindsight and after the fact.

It is now a fact that humans do not follow any of the precepts of so called axiomatic rationality. $\mathrm{Hu}-$ mans are fundamentally 'irrational' vis-à-vis the artificial axioms of rationality. For example, notions of transitivity are simplifying artificial constructs which do not and cannot hold in the real world of human decision making. Human decisions continue to be far superior to anything recommended by the pre-scientific artifacts of expected utility maximization, goals satisficing or maximization with respect to 'given' constraints.

With the advances of decision support systems, the decision maker is being properly aided in the very process of decision production through repeated reformulations of the decisional network. Humans create or construct both information and decisions. All important aspects of decision making: criteria, alternatives, representations and evaluations are maintained in a constructive flux of mutual adjustment and interdependent co-determination. Nothing is to be fixed a priori.

Human decision-making process is a complex, organizationally closed search for internal consistency, passing through interrelated layers of definitions and redefinitions of the problem. According to Nappelbaum [3], a problem has been fully formulated only after it has been solved. All aspects of decision making are changing and mutually adjusting until a relatively stable pattern or cognitive equilibrium among them has been reached [4].

The problem is thus dissolved, the harmony achieved and recognized, there remains no 'choice' possibility other than that of the (socially) approved pattern (ideal solution, dominant option, prominent alternative). Only then, retrospectively, one could look back and say: 'I have decided ...'

Nappelbaum [3] bases his choice-producing networks on the interconnections between the respective languages: of (1) option descriptions, (2) instrumental intentions and (3) value judgments. Decision making thus cannot be separated from the production of knowledge and therefore from the construction of individual local worlds. Any 'large' world (universe) consists of a variety of cognitively closed and essentially unmergeable 'small' local worlds: a multiverse.

It is quite different to interact with the decision maker for the purposes of forcing him into a priori fixed formulas, patterns or contexts (such as 'racing' him back and forth and around through a fixed set of nondominated solutions) and to guide him through his own creative search process. Decision making is a process of continuous reformulation of the problem [4].

Following Fuller [2], knowledge production can be viewed from two essential vantage points of knowledge-production designer:

1) Assuming that he already knows the purpose of producing knowledge, he can then determine how and whether the parts of the knowledge production process function to realize that purpose. This is the view of classical epistemology.

2) Assuming that he already knows that the parts of the knowledge production process function optimally to realise some purpose, he can determine what that purpose could and could not be. This is panglossian design: knowledge production process works optimally towards some ends, but it is matter of empirical determination what the ends are. What sorts of goals can be realized given the actual structural constraints on knowledge production? This is one of the fundamental questions of the CE paradigm. 
Surprisingly, and somewhat counter intuitively, the actual knowledge production processes lack any clear indicators for such qualities as retention, accumulation, and convergence. The design of effective decision support systems should therefore become affected in a profound way.

Under the CE paradigm, each problem must be initially ill-structured. Yet, each solution is the solution to a well-structured problem, i.e., problem in cognitive equilibrium. So, each ill-structured problem is a problem well-structured towards its cognitive equilibrium.

Criteria or attributes are not objective properties of $X$ which can be captured, measured and encapsulated in a utility or membership function. The meaning of attribute tall is not fixed and it cannot be mapped onto some numerical scale of measured height. The meaning of tall is negotiated, over and over, in different social contexts and can be related to all or any portion of the scale.

There is therefore a sort of cognitive economy of a community of knowledge negotiating agents: objects are regularly passed from being represented to being representants, back and forth, so as to maintain cognitive equilibrium. The word table is not as clear and distinct a representation of the table as the table itself. Balancing of representational gains and losses is equilibrium in a cognitive economy.

One person's circumstance - purposes, intentions, experiences, thoughts, concepts, sensations, emotions - cannot be directly compared with another person's circumstance. There is no 'true picture' of the world 'as is.'

The only thing we can hope for is a revealed compatibility or acceptability of meaning in a given context. This can only be revealed via communication or conservation, it can only be negotiated. People will engage in such negotiation only if they intend to cooperate, if they wish to coordinate their action. So, in order to study fuzziness and ambiguity of human language we have to study human cooperation in coordinating their task, labor and knowledge activities.

One of the reasons why humans choose to employ one or another fuzzy label (even if precise designation is available) is to propose, define, reframe or impose a particular context within which coordination of action (cooperation or com- petition) is to take place. A fuzzy label thus allows extension of meaning, induces overlaps, intersections or unions of contexts.

To think that the truth of the whole implies the truth of its historically constituent parts is to commit the fallacy of division. Recall that free market functions to the benefit of all participants but its participants intend to benefit only themselves.

Given current corpus of knowledge, design the most efficient division of cognitive labor that would have produced the corpus. How different would your design be from the disciplinary boundaries drawn in the actual course of history? Problem of bureaucratic management. Are disciplinary boundaries in principle dispensable? Disciplines often cross-classify the same subject matter and impede any mutually useful synthesis.

Modeling implications of the CE paradigm are undoubtedly rich, challenging and far-reaching. However, its philosophical implications are even more challenging:

For the first time in history we are posed to understand decision making not merely as computation of the world given 'out there,' but as the very way of constructing our local world, ordering our individual and collective experience, making sense of the 'chaos' of reality. Making decisions does not mean finding our ways through a fixed maze (problem solving) - decision making refers to the very construction of that maze - ordering of nature so that we ourselves can find our way through it ...

The first CE-paradigm research conference ('Decision Making As Organization of Knowledge') is going to take place during the 8th International Congress of Cybernetics and Systems, New York City, June 11-15, 1990. In the mean time, the International Institute for Advanced Systems Analysis (IIASA) and Japanese cognitive scientists are planning introductory meetings of active CE-researchers. Human Systems Management, as always, is going to be there, at the source.

\section{References}

[1] Margolis, H., Patterns, Thinking, and Cognition, University of Chicago Press, Chicago, 1988. 
[2] Fuller, S., Social Epistemology, Indiana University Press, Bloomington, 1988.

[3] Nappelbaum, E., 'Logic of Problem Formulation and Choice,' Journal of Applied Systems Analysis, (in press) 1989.

[4] Zeleny, M., 'Multicriteria Decision Making,' in: Systems \& Control Encyclopedia, 1st Supplement, Pergamon Press, Elmsford, N.Y., 1989.

[5] Zeleny, M., Multiple Criteria Decision Making, McGrawHill, New York, 1982, pp. 87-92.
[6] Zeleny, M., (ed.), Autopoiesis: A Theory of Living Organization, North Holland, New York, 1981.

Milan ZELENY

Graduate School of Business

Fordham University at Lincoln Center

New York, NY 10023

U.S.A. 